\title{
Compositional Verification of Large-Scale Stochastic Systems via Relaxed Small-Gain Conditions
}

\author{
Abolfazl Lavaei, Student Member, IEEE, and Majid Zamani, Senior Member, IEEE
}

\begin{abstract}
In this paper, we provide a compositional framework for the construction of finite abstractions (a.k.a. finite Markov chains (MCs)) for networks of not necessarily stable discrete-time stochastic systems. The proposed scheme is based on a notion of finite-step stochastic simulation functions, using which one can employ an abstract system as a substitution of the original one in the verification process with guaranteed error bounds. To this end, we first develop a new type of smallgain conditions which are less conservative than the existing ones in compositionally quantifying the probabilistic distance between the interconnection of stochastic subsystems and that of their finite abstractions. We then propose an approach to construct finite MCs together with their corresponding finitestep simulation functions for discrete-time nonlinear stochastic systems satisfying a finite-step version of an incremental inputto-state stability ( $\delta$-ISS) property. We also construct finite MCs for a particular class of nonlinear stochastic systems whose 1 -step $\delta$-ISS can be easily verified using the semidefinite programming. Finally, we demonstrate the effectiveness of the proposed results to a network of four subsystems such that one of them is not stable.
\end{abstract}

\section{INTRODUCTION}

Motivations. Large-scale stochastic systems play significant roles in many real-life applications including power grids, traffic networks, and so on. Since analyzing these large-scale systems is inherently very challenging, one promising approach is to first abstract the original system by a simpler one (with finite-state set), perform analysis over the abstract model, and finally carry the analysis back over the concrete system, by providing guaranteed error bounds.

Unfortunately, construction of finite MCs for large-scale stochastic systems in a monolithic manner suffers severely from the curse of dimensionality: the computational complexity of constructing finite MCs grows exponentially w.r.t. the number of state variables. To mitigate this issue, one promising solution is to consider the large-scale system as an interconnected system composed of several smaller subsystems, and provide a compositional framework for the construction of finite MCs for the given system using finite MCs of smaller subsystems.

Related Literature. There have been several results on the construction of finite abstractions for stochastic systems. Construction of finite MCs for formal verification is initially proposed in [1] and extended in [2] with an available toolbox [3]. Finite bisimilar abstractions (a.k.a. labeled transition systems) for randomly switched stochastic systems are presented in [4]. Moreover, finite bisimilar abstractions for in-

This work was supported in part by the H2020 ERC Starting Grant AutoCPS (grant agreement No. 804639).

A. Lavaei is with the Department of Electrical and Computer Engineering, Technical University of Munich, Germany. M. Zamani is with the Computer Science Department, University of Colorado Boulder, USA. M. Zamani is with the Computer Science Department, Ludwig Maximilian University of Munich, Germany. Email: lavaei@tum.de, majid.zamani@colorado.edu. crementally stable stochastic switched systems are discussed in [5]. Compositional construction of finite abstractions using dynamic Bayesian networks, dissipativity-type reasoning and small-gain conditions are respectively proposed in [6], [7], and [8]. Compositional infinite and finite abstractions in a unified framework via approximate probabilistic relations are proposed in [9], [10]. Compositional construction of finite MDPs for large-scale stochastic switched systems via a dissipativity approach is presented in [11]. An (in)finite abstraction technique for synthesis of stochastic control systems is recently discussed in [12].

In the context of stability verification, nonconservative small-gain conditions based on finite-step Lyapunov functions were originally introduced in [13]. Moreover, nonconservative small-gain conditions for closed sets using finitestep ISS Lyapunov functions are presented in [14]. Recently, compositional construction of finite abstractions via relaxed small-gain conditions for discrete-time non-stochastic systems is proposed in [15]. Although the proposed results in [15] employ relaxed small-gain conditions, their setting is non-stochastic and the closeness guarantee is not established at each instant of time.

Our Contributions. In this paper, we develop a compositional scheme using relaxed small-gain type conditions for the construction of finite MCs for networks of discrete-time stochastic systems that are not necessarily stable. The proposed framework relies on a relation between each subsystem and its finite MC employing a notion of finite-step stochastic pseudo-simulation functions. This relaxation results in a less conservative version of small-gain conditions, using which one can compositionally construct finite MCs such that stability of each subsystem is not necessarily required. We also provide the probabilistic closeness guarantee between the interconnected stochastic systems and that of their finite MCs for the whole state trajectory. We quantify that if the state discretization parameter is small enough, the sampled Markov chain will be close enough to the original system. Proofs of all statements are omitted due to space limitations.

Recent Works. Compositional construction of finite abstractions for networks of discrete-time stochastic systems is recently discussed in [16], but by employing a different compositionality scheme based on dissipativity theory. Our proposed approach here differs from the one in [16] in three main directions. First and foremost, the proposed compositional synthesis approach here is less conservative than the one proposed in [16] since the overall approximation error here is computed based on the maximum error of subsystems instead of their linear combinations which is the case in [16]. Second, although the provided results in [16] do not ask the individual subsystems to be stable, our probabilistic closeness guarantee here is more general 
than the one proposed in [16] since we provide the closeness guarantee for the whole state trajectory, while [16] quantifies the error only at some specific steps without providing the closeness for all time steps. As our last main contribution, we enlarge the class of systems for the construction of finite MCs by adding nonlinearities to the dynamics satisfying an incremental quadratic inequality (cf. (26)), whereas the provided results in [16] only handle the class of nonlinearities satisfying a slope restriction (cf. [16, Subsection 5.2]).

Compositional construction of finite abstractions for networks of stochastic systems via a relaxed small-gain condition is recently discussed in [17]. Our proposed approach differs from the one in [17] in two key directions. First, we provide the closeness guarantee for the whole state trajectory while [17] quantifies the error only at some specific time steps. Second, we propose an abstract-construction scheme for the general setting of nonlinear stochastic systems and also a particular class of nonlinear systems whose nonlinearities satisfy an incremental quadratic inequality, whereas the construction framework in [17] only handles linear systems.

\section{A. Notation}

\section{Discrete-Time Stochastic Systems}

The sets of real numbers, nonnegative and positive integers are denoted by $\mathbb{R}, \mathbb{N}:=\{0,1,2, \ldots\}$, and $\mathbb{N}_{\geq 1}:=$ $\{1,2,3, \ldots\}$, respectively. We employ $x=\left[x_{1} ; \ldots ; x_{N}\right]$ to denote the corresponding vector of dimension $\sum_{i} n_{i}$, given $N$ vectors $x_{i} \in \mathbb{R}^{n_{i}}, n_{i} \in \mathbb{N}_{\geq 1}, i \in\{1, \ldots, N\}$. We denote by $\|\cdot\|$ and $\|\cdot\|_{2}$ the infinity and Euclidean norm, respectively. The identity matrix in $\mathbb{R}^{n \times n}$ and the column vector in $\mathbb{R}^{n \times 1}$ with all elements equal to one are denoted by $\mathbb{I}_{n}$, and $\mathbb{1}_{n}$, respectively. We denote by $\mathcal{I}_{d}$ and symbol $\circ$, the identity function and composition of functions, respectively. A function $\gamma: \mathbb{R}_{\geq 0} \rightarrow \mathbb{R}_{\geq 0}$, is said to be a class $\mathcal{K}$ function if it is continuous, strictly increasing, and $\gamma(0)=0$. A class $\mathcal{K}$ function $\gamma(\cdot)$ is said to be a class $\mathcal{K}_{\infty}$ if $\gamma(r) \rightarrow \infty$ as $r \rightarrow \infty$.

\section{B. Discrete-Time Stochastic Systems}

Definition 2.1: A discrete-time stochastic system (dt-SS) in this work is characterized by the tuple

$$
\Sigma=(X, W, \varsigma, f)
$$

where:

- $X \subseteq \mathbb{R}^{n}$ is a Borel space as the state space of the system. We denote by $(X, \mathcal{B}(X))$ the measurable space with $\mathcal{B}(X)$ being the Borel sigma-algebra on the state space;

- $W \subseteq \mathbb{R}^{p}$ is a Borel space as the internal input space of the system;

- $\varsigma$ is a sequence of independent and identically distributed (i.i.d.) random variables from a sample space $\Omega$ to the set $V_{\varsigma}$

$$
\varsigma:=\left\{\varsigma(k): \Omega \rightarrow V_{\varsigma}, k \in \mathbb{N}\right\},
$$

- $f: X \times W \times V_{\varsigma} \rightarrow X$ is a measurable function characterizing the state evolution of the system.

For given initial state $x(0) \in X$ and input sequence $\{\mathrm{w}(k)$ : $\Omega \rightarrow W, k \in \mathbb{N}\}$, the evolution of the state of dt-SS $\Sigma$ in (1) can be described by

$$
\Sigma:\left\{\begin{array}{l}
x(k+1)=f(x(k), \mathrm{w}(k), \varsigma(k)), \\
x(\cdot) \in X, \mathrm{w}(\cdot) \in W, k \in \mathbb{N} .
\end{array}\right.
$$

The set $\mathcal{W}$, associated to $W$, is a collection of sequences $\{\mathrm{w}(k): \Omega \rightarrow W, k \in \mathbb{N}\}$, in which $\mathrm{w}(k)$ is independent of $\varsigma(t)$ for any $k, t \in \mathbb{N}$ and $t \geq k$. For any initial state $a \in X$, and $\mathrm{w}(\cdot) \in \mathcal{W}$, the random sequence $x_{a \mathrm{w}}: \Omega \times \mathbb{N} \rightarrow X$, satisfying (2) is called the solution process of $\Sigma$ under the initial state $a$, and the internal input w. If $X, W$ are finite sets, system $\Sigma$ is called finite, and infinite otherwise.

In the following subsection, we define the $M$-sampled systems, based on which one can employ the proposed finitestep stochastic simulation function to quantify the mismatch between the interconnected dt-SS and that of their (in)finite abstractions.

\section{M-Sampled Systems}

In general, the stability requirement for individual subsystems in [7], [8], [18], [19], [20] may not hold even if the interconnected system is stable. We show that this requirement can be relaxed by incorporating the stabilizing effect of the neighboring subsystems in a local unstable subsystem. Once the stability effect is appeared, we construct finite MCs of subsystems and employ a small-gain type condition to provide the compositionality results. Our approach relies on looking at the solution process of the system in future time instances while incorporating the interconnection of subsystems. The following example illustrates this idea.

Example 2.2: Consider two linear dt-SS $\Sigma_{1}, \Sigma_{2}$ with dynamics

$$
\begin{aligned}
& x_{1}(k+1)=1.01 x_{1}(k)+0.4 \mathrm{w}_{1}(k)+\varsigma_{1}(k), \\
& x_{2}(k+1)=0.55 x_{2}(k)-0.2 \mathrm{w}_{2}(k)+\varsigma_{2}(k),
\end{aligned}
$$

that are connected with the constraint $\mathrm{w}_{i}=x_{3-i}$, for $i=$ $\{1,2\}$. Note that the first subsystem is not stable. By looking at the solution process two steps ahead and considering the interconnection, one can rewrite

$$
\begin{aligned}
x_{1}(k+2)= & 0.94 x_{1}(k)+0.62 w_{1}(k)+0.4 \varsigma_{2}(k) \\
& +1.01 \varsigma_{1}(k)+\varsigma_{1}(k+1), \\
x_{2}(k+2)= & 0.22 x_{2}(k)-0.31 w_{2}(k)-0.2 \varsigma_{1}(k) \\
& +0.55 \varsigma_{2}(k)+\varsigma_{2}(k+1),
\end{aligned}
$$

which we denote them by $\Sigma_{\mathrm{aux} 1}, \Sigma_{\mathrm{aux} 2}$, in which $w_{i}=$ $x_{3-i}$, for $i=\{1,2\}$. These two subsystems in (4) are now stable. This motivates us to construct abstractions of original subsystems (3) based on auxiliary subsystems (4).

Remark 2.3: Note that after interconnecting the subsystems to each other and propagating the dynamics in the next $M$-steps, the interconnection topology may change (cf. case study). Then the internal input of the auxiliary system (i.e., $w$ ) is different from the original one (i.e., w).

Next lemma shows how dynamics of the $M$-sampled systems, call auxiliary system $\Sigma_{\text {aux }}$, can be acquired.

Lemma 2.4: Suppose we are given $N$ dt-SS $\Sigma_{i}$ defined by

$$
\Sigma_{i}:\left\{\begin{array}{l}
x_{i}(k+1)=f_{i}\left(x_{i}(k), \mathrm{w}_{i}(k), \varsigma_{i}(k)\right), \\
x_{i}(\cdot) \in X_{i}, \mathbf{w}_{i}(\cdot) \in W_{i}, k \in \mathbb{N},
\end{array}\right.
$$

which are connected in a network with constraints $\mathrm{w}_{i}=$ $\left[x_{1} ; \ldots ; x_{i-1} ; x_{i+1} ; \ldots ; x_{N}\right], \forall i \in\{1, \cdots, N\}$. The $M-$ sampled systems $\Sigma_{\text {auxi } i}$, which are the solutions of $\Sigma_{i}$ at time instances $k=j M, j \in \mathbb{N}$, have the dynamics

$$
\Sigma_{\text {aux } i}:\left\{\begin{array}{l}
x_{i}(k+M)=\tilde{f}_{i}\left(x_{i}(k), w_{i}(k), \tilde{\varsigma}_{i}(k)\right), \\
x_{i}(\cdot) \in X_{i}, w_{i}(\cdot) \in \tilde{W}_{i}, k=j M, j \in \mathbb{N},
\end{array}\right.
$$

where $\tilde{\varsigma}_{i}(k)$ is a vector containing noise terms as follows:

$$
\begin{aligned}
& \tilde{\varsigma}_{i}(k)=\left[\bar{\varsigma}_{1}(k) ; \ldots ; \bar{\varsigma}_{i}^{*}(k) ; \ldots ; \bar{\varsigma}_{N}(k)\right], \bar{\varsigma}_{i}^{*}(k)=\left[\varsigma_{i}(k) ; \ldots ;\right. \\
& \left.\varsigma_{i}(k+M-1)\right], \bar{\varsigma}_{j}(k)=\left[\varsigma_{j}(k) ; \ldots ; \varsigma_{j}(k+M-2)\right], \\
& \forall j \in\{1, \ldots N\}, j \neq i .
\end{aligned}
$$

Note that some of the noise term in $\tilde{\varsigma}_{i}(k)$ may be omitted depending on the interconnection graph, but all the terms 
are present for a fully interconnected network. Proof of Lemma 2.4 is based on recursive application of the vector field $f_{i}$. We refer the interested readers to [16, Example 2.6] for a detailed computation of vector field $\tilde{f}_{i}$ on a network of two linear dt-SS.

\section{Finite-Step Stochastic Pseudo-Simulation AND Simulation FunCtions}

In this section, we first define a notion of finite-step stochastic pseudo-simulation functions inspired by the notion of finite-step Lyapunov functions [21].

Definition 3.1: Consider dt-SS $\Sigma_{i}$ and $\widehat{\Sigma}_{i}$, where $\hat{W}_{i} \subseteq$ $W_{i}$ and $\hat{X}_{i} \subseteq X_{i}$. A function $S_{i}: X_{i} \times \hat{X}_{i} \rightarrow \mathbb{R}_{>0}$ is called a finite-step stochastic pseudo-simulation function (FPSF) from $\widehat{\Sigma}_{i}$ to $\Sigma_{i}$ if there exist $M \in \mathbb{N}_{\geq 1}, \alpha_{i}, \kappa_{i} \in \mathcal{K}_{\infty}$ with $\kappa_{i}<\mathcal{I}_{d}, \rho_{\text {int } i} \in \mathcal{K}_{\infty} \cup\{0\}$, and constant $\psi_{i} \in \mathbb{R}_{\geq 0}$, such that for all $k=j M, j \in \mathbb{N}$,

$$
\begin{aligned}
& \text { - } \forall x_{i}:=x_{i}(k) \in X_{i}, \hat{x}_{i}:=\hat{x}_{i}(k) \in \hat{X}_{i} \text {, } \\
& \alpha_{i}\left(\left\|x_{i}-\hat{x}_{i}\right\|\right) \leq S_{i}\left(x_{i}, \hat{x}_{i}\right),
\end{aligned}
$$

- $\forall x_{i}:=x_{i}(k) \in X_{i}, \hat{x}_{i}:=\hat{x}_{i}(k) \in \hat{X}_{i}, \forall w_{i}:=w_{i}(k) \in \tilde{W}_{i}$, $\forall \hat{w}_{i}:=\hat{w}_{i}(k) \in \hat{W}_{i}$,

$$
\begin{aligned}
& \mathbb{E}\left[S_{i}\left(x_{i}(k+M), \hat{x}_{i}(k+M)\right) \mid x_{i}, \hat{x}_{i}, w_{i}, \hat{w}_{i}\right] \\
& \leq \max \left\{\kappa_{i}\left(S_{i}\left(x_{i}, \hat{x}_{i}\right)\right), \rho_{\text {int } i}\left(\left\|w_{i}-\hat{w}_{i}\right\|\right), \psi_{i}\right\} .
\end{aligned}
$$

We call $\widehat{\Sigma}_{i}$ an abstraction of $\Sigma_{i}$ if there exists an FPSF $S_{i}$ from $\widehat{\Sigma}_{i}$ to $\Sigma_{i}$. We drop the term finite-step for the case $M=1$, and instead call it a classic pseudo-simulation function ([7], [8]).

Remark 3.2: Note that $\kappa_{i}$ in (9) depends implicitly on $M$ and is required to be less than $\mathcal{I}_{d}$. Hence, the proposed FPSF $S_{i}$ here is less conservative than the conventional simulation functions (where $M=1$ ) defined in [7], [8]. In other words, condition (9) may not be satisfied for $M=1$ but may hold for some $M \in \mathbb{N}_{>1}$ which is the case in this work. Such an implicit dependency on $M$ will increase the class of systems for which condition (9) is satisfiable since this relaxation allows some of the individual subsystems to be even unstable (cf. case study).

Definition 3.1 can also be stated for systems without internal inputs by omitting all the terms related to $w, \hat{w}$ as in the following definition. Such systems can be acquired by interconnecting the subsystems.

Definition 3.3: Consider two dt-SS $\Sigma$ and $\widehat{\Sigma}$ without internal input, where $\hat{X} \subseteq X$. A function $V: X \times \hat{X} \rightarrow \mathbb{R}_{>0}$ is called a finite-step stochastic simulation function (FSF) from $\widehat{\Sigma}$ to $\Sigma$ if there exists $M \in \mathbb{N}_{\geq 1}$, and $\alpha \in \mathcal{K}_{\infty}$ such that

$$
\begin{gathered}
\forall x:=x(k) \in X, \hat{x}:=\hat{x}(k) \in \hat{X} \\
\alpha(\|x-\hat{x}\|) \leq V(x, \hat{x}), \\
\text { - } \forall x:=x(k) \in X, \hat{x}:=\hat{x}(k) \in \hat{X}, \\
\mathbb{E}[V(x(k+M), \hat{x}(k+M)) \mid x, \hat{x}] \leq \max \{\kappa(V(x, \hat{x})), \psi\},
\end{gathered}
$$
N.

for some $\kappa \in \mathcal{K}_{\infty}$ with $\kappa<\mathcal{I}_{d}, \psi \in \mathbb{R}_{\geq 0}$, and $k=j M, j \in$

In order to show the usefulness of SSF in comparing the state trajectories of two dt-SS (without internal inputs), we need the following technical lemma borrowed from [19, Theorem 3.3]. This Lemma holds for our setting here since our max form implies the additive form employed in [19, Theorem 3.3].
Lemma 3.4: Let $\Sigma$ and $\widehat{\Sigma}$ be two dt-SS without internal inputs, where $\hat{X} \subseteq X$. Suppose $V$ is an FSF from $\Sigma$ to $\Sigma$ at times $k=j M, j \in \mathbb{N}$, and there exists a constant $0<\hat{\kappa}<1$ such that the function $\kappa \in \mathcal{K}_{\infty}$ in (11) satisfies $\kappa(r) \geq \hat{\kappa} r$, $\forall r \in \mathbb{R}_{>0}$. For any random variables $a$ and $\hat{a}$ as the initial states of the two dt-SS, the following inequality holds

$$
\begin{aligned}
& \mathbb{P}\left\{\sup _{k=j M, 0 \leq j \leq T_{d}}\left\|x_{a}(k)-\hat{x}_{\hat{a}}(k)\right\| \geq \varepsilon \mid[a ; \hat{a}]\right\} \\
& \leq \begin{cases}1-\left(1-\frac{V(a, \hat{a})}{\alpha(\varepsilon)}\right)\left(1-\frac{\psi}{\alpha(\varepsilon)}\right)^{T_{d}} & \text { if } \alpha(\varepsilon) \geq \frac{\psi}{\hat{\kappa}}, \\
\left(\frac{V(a, \hat{a})}{\alpha(\varepsilon)}\right)(1-\hat{\kappa})^{T_{d}}+\left(\frac{\psi}{\hat{\kappa} \alpha(\varepsilon)}\right)\left(1-(1-\hat{\kappa})^{T_{d}}\right) & \text { if } \alpha(\varepsilon)<\frac{\psi}{\hat{\kappa}} .\end{cases}
\end{aligned}
$$

In the next subsection, we provide the closeness guarantee for all time instances by utilizing Lemma 3.4

\section{A. Closeness Guarantee for all Time Instances}

Suppose we are given an interconnected network composed of $N$ stochastic subsystems $\Sigma_{i}$ as in (5), with the interconnection constraint $\mathrm{w}_{i j}=x_{j}, \forall i, j \in\{1, \ldots, N\}, i \neq$ $j$. The $M$-sampled systems for all time instances contain $M$ different dynamics starting from initial values $\left\{x_{i}(0), x_{i}(1), \ldots, x_{i}(M-1)\right\}$ as follows:

$$
\begin{aligned}
& x_{i}(k+M)=\tilde{f}_{i}\left(x_{i}(k), w_{i}(k), \tilde{\varsigma}_{i}(k)\right), \\
& x_{i}(k+M+1)=\tilde{f}_{i}\left(x_{i}(k+1), w_{i}(k+1), \tilde{\varsigma}_{i}(k+1)\right),
\end{aligned}
$$

$$
x_{i}(k+2 M-1)=\tilde{f}_{i}\left(x_{i}(k+M-1), w_{i}(k+M-1), \tilde{\varsigma}_{i}(k+M-1)\right),
$$

where $k=j M, j \in \mathbb{N}$. In order to show the closeness of two interconnected dt-SS for all time instants using Lemma 3.4, we also require the following assumption.

Assumption 1: Assume that there exist $\mathcal{K}_{\infty}$ and concave function $\mu$ and constant $\theta \geq 0$ such that for all $x(k):=x \in$ $X$ and $\hat{x}(k):=\hat{x} \in \hat{X}$,

$$
\mathbb{E}[V(x(k+1), \hat{x}(k+1)) \mid x, \hat{x}] \leq \mu(V(x, \hat{x}))+\theta .
$$

Remark 3.5: Note that Assumption 1 is a standard one employed in the definition of classic stochastic simulation functions $(M=1)$ similar to the one appeared in [19, Definition 3.2]. Remark that Assumption 1 is less restrictive than the condition (11) since we do not require $\mu<\mathcal{I}_{d}$ (cf. case study).

Next theorem shows the closeness of two interconnected dtSS for all time instants.

Theorem 3.6: Let $\Sigma$ and $\widehat{\Sigma}$ be two dt-SS without internal inputs (i.e. $\rho_{\text {int }}(\cdot) \equiv 0$ ), where $X \subseteq X$. Suppose $V$ is an FSF from $\widehat{\Sigma}$ to $\Sigma$ at times $k=j M, j \in \mathbb{N}$, and Assumption 1 holds. For any random variables $a$ and $\hat{a}$ as the initial states of the two dt-SS, closeness of the two interconnected systems for all time instants within the time horizon $T_{d} M$ can be acquired as

$$
\begin{aligned}
& \mathbb{P} \begin{cases}\left.\sup _{k \in \mathbb{N}, 0 \leq k \leq T_{d} M}\left\|x_{a}(k)-\hat{x}_{\hat{a}}(k)\right\| \geq \varepsilon \mid[a ; \hat{a}]\right\} & \text { if } M=1,\end{cases} \\
& \leq \begin{cases}\eta_{0}+\beta_{0} V(a, \hat{a}) & \text { if } M \geq 2, \\
\sum_{i=0}^{M-1}\left(\eta_{i}+\beta_{i} \mu^{i}(V(a, \hat{a}))+\beta_{i} \sum_{n=0}^{i-1} \mu^{n}(\theta)\right) & \end{cases}
\end{aligned}
$$

where $\mu^{0}=\mathcal{I}_{d}$, and $\eta_{i}, \beta_{i}$ are some constants coming from the right-hand side of (12) in the form of $\eta_{i}+\beta_{i} V(a, \hat{a})$,

with
$\left\{\begin{array}{l}\eta_{i}=1-\left(1-\frac{\psi_{i}}{\alpha_{i}(\varepsilon)}\right)^{T_{d}}, \beta_{i}=\frac{1}{\alpha_{i}(\varepsilon)}\left(1-\frac{\psi_{i}}{\alpha_{i}(\varepsilon)}\right)^{T_{d}} \quad \text { if } \alpha_{i}(\varepsilon) \geq \frac{\psi_{i}}{\hat{\kappa}_{i}}, \\ \eta_{i}=\frac{\psi_{i}}{\hat{\kappa}_{i} \alpha_{i}(\varepsilon)}\left(1-\left(1-\hat{\kappa}_{i}\right)^{T_{d}}\right), \beta_{i}=\frac{1}{\alpha_{i}(\varepsilon)}\left(1-\hat{\kappa}_{i}\right)^{T_{d}} \quad \text { otherwise. }\end{array}\right.$

To show Theorem 3.6, we respectively employed Boole's inequality, law of total expectation, Assumption 1, Jensen's 
inequality for concave function $\mu$, induction step for $i$, and subadditivity of $\mu$. Note that $\mu$ is indeed subadditive because it is concave and $\mu:[0, \infty] \rightarrow[0, \infty]$ with $\mu(0)=0[22$, Chapter 12].

\section{Compositional Abstractions FOR INTERCONNECTED SYSTEMS}

In this section, we assume that we are given a complex stochastic system $\Sigma$ composed of $N \in \mathbb{N}_{>1}$ discrete-time stochastic subsystems $\Sigma_{i}$ as in (5), where their internal inputs are partitioned as

$$
\mathrm{w}_{i}=\left[\mathrm{w}_{i 1} ; \ldots ; \mathrm{w}_{i(i-1)} ; \mathrm{w}_{i(i+1)} ; \ldots ; \mathrm{w}_{i N}\right] .
$$

Now we are ready to define the interconnected stochastic systems.

Definition 4.1: Suppose we are given $N \in \mathbb{N}_{\geq 1}$ discretetime stochastic subsystems $\Sigma_{i}, i \in\{1, \ldots, N\}$, with the internal input configuration as in (15). The interconnection of $\Sigma_{i}$ for any $i \in\{1, \ldots, N\}$, denoted by $\mathcal{I}\left(\Sigma_{1}, \ldots, \Sigma_{N}\right)$, is the interconnected stochastic system $\Sigma$, such that $X:=$ $\prod_{i=1}^{N} X_{i}$, and function $f:=\prod_{i=1}^{N} f_{i}$, subjected to the following constraint:

$$
\forall i, j \in\{1, \ldots, N\}, i \neq j: \quad \mathrm{w}_{i j}=x_{j}, \quad X_{j} \subseteq W_{i j} .
$$

Prior to presenting the next theorem, we raise the following small-gain assumption.

Assumption 2: Assume that $\mathcal{K}_{\infty}$ functions $\kappa_{i j}$ defined as

$$
\kappa_{i j}(r):= \begin{cases}\kappa_{i}(r) & \text { if } i=j \\ \rho_{\operatorname{int} i}\left(\alpha_{j}^{-1}(r)\right) & \text { if } i \neq j,\end{cases}
$$

satisfy

$$
\kappa_{i_{1} i_{2}} \circ \kappa_{i_{2} i_{3}} \circ \cdots \circ \kappa_{i_{r-1} i_{r}} \circ \kappa_{i_{r} i_{1}}<\mathcal{I}_{d}
$$

for all sequences $\left(i_{1}, \ldots, i_{r}\right) \in\{1, \ldots, N\}^{r}$ and $r \in$ $\{1, \ldots, N\}$, where $\alpha_{j}, \kappa_{i}$, and $\rho_{\text {int } i}$ are the functions appeared in Definition 3.1. The small-gain condition (17) implies the existence of $\mathcal{K}_{\infty}$ functions $\sigma_{i}>0$ [23, Theorem 5.5], satisfying

$$
\max _{i, j}\left\{\sigma_{i}^{-1} \circ \kappa_{i j} \circ \sigma_{j}\right\}<\mathcal{I}_{d}, \quad i, j=\{1, \ldots, N\} .
$$

In the next theorem, we leverage the small-gain Assumption 2 together with concavity assumption of $\max _{i} \sigma_{i}^{-1}$ to show the main compositionality result of the paper.

Theorem 4.2: Suppose we are given the interconnected dtSS $\Sigma=\mathcal{I}\left(\Sigma_{1}, \ldots, \Sigma_{N}\right)$ induced by $N \in \mathbb{N}_{\geq 1}$ stochastic subsystems $\Sigma_{i}$. Let each $\Sigma_{i}$ admits an abstraction $\widehat{\Sigma}_{i}$ with the corresponding FPSF $S_{i}$. If Assumption 2 holds and also

$$
\forall i, j \in\{1, \ldots, N\}, i \neq j: \quad \hat{\mathrm{w}}_{\mathrm{ij}}=\hat{x}_{j}, \quad \hat{X}_{j} \subseteq \hat{W}_{i j},
$$

then function $V(x, \hat{x})$ defined as

$$
V(x, \hat{x}):=\max _{i}\left\{\sigma_{i}^{-1}\left(S_{i}\left(x_{i}, \hat{x}_{i}\right)\right)\right\}
$$

for $\sigma_{i}$ as in (18), is an FSF function from $\widehat{\Sigma}=$ $\mathcal{I}\left(\widehat{\Sigma}_{1}, \ldots, \widehat{\Sigma}_{N}\right)$ to $\Sigma=\mathcal{I}\left(\Sigma_{1}, \ldots, \Sigma_{N}\right)$ at times $k=$ $j M, j \in \mathbb{N}$ provided that $\max _{i} \sigma_{i}^{-1}$ is concave.

\section{Construction of Finite Markov Chains}

Here, we first discuss the construction of finite MCs as abstractions of dt-SS in Subsection V-A. We then propose an approach to construct finite MCs for the general setting of discrete-time nonlinear stochastic systems satisfying an incremental ISS property in Subsection V-B. Finally, we focus on a particular class of discrete-time nonlinear stochastic systems $\Sigma_{i}$ by providing an approach on the construction of their classic pseudo-simulation functions $(M=1)$ in Subsection V-C.

\section{A. Finite Markov Chains of $d t-S S$}

A dt-SS $\Sigma_{\text {aux }}$ in (6) can be equivalently represented as a Markov Chain [24]

$$
\Sigma=\left(X, \tilde{W}, T_{\times}\right)
$$

where $T_{\times}: \mathcal{B}(X) \times X \times \tilde{W} \rightarrow[0,1]$, is a conditional stochastic kernel that assigns to any $x \in X$, and $w \in \tilde{W}$ a probability measure $T_{\mathrm{x}}(\cdot \mid x, w)$ on the measurable space $(X, \mathcal{B}(X))$ such that for any set $\mathcal{A} \in \mathcal{B}(X)$,

$$
\mathbb{P}(x(k+1) \in \mathcal{A} \mid x(k), w(k))=\int_{\mathcal{A}} T_{\times}(d x(k+1) \mid x(k), w(k)) .
$$

For given $w(\cdot)$, the stochastic kernel $T_{\mathrm{x}}$ captures the evolution of the state of $\Sigma_{\text {aux }}$ and can be uniquely determined by the pair $(\tilde{\varsigma}, \tilde{f})$ using $(6)$.

Given a dt-SS $\Sigma_{\text {aux }}$ in (6), we construct its finite MC $\widehat{\Sigma}_{\text {aux }}$ as the finite abstraction of the auxiliary system following the approach of [2]. The abstraction algorithm works based on selecting finite partitions of state and input sets as

$$
X=\cup_{i=1}^{n_{x}} \mathrm{X}_{i}, \quad \tilde{W}=\cup_{i=1}^{n_{w}} \tilde{\mathrm{W}}_{i},
$$

and selecting representative points $\bar{x}_{i} \in \mathrm{X}_{i}$, and $\bar{w}_{i} \in \tilde{\mathbf{W}}_{i}$. It then constructs finite sets $\hat{X}=\left\{\bar{x}_{i}, i=1, \ldots, n_{x}\right\}$, and $\hat{W}=\left\{\bar{w}_{i}, i=1, \ldots, n_{w}\right\}$, that contain representative points of the partition sets as abstract states and inputs. Dynamics over the abstract states are defined via function $\hat{f}: \hat{X} \times \hat{W} \times$ $\times V_{\varsigma} \rightarrow \hat{X}$ as

$$
\hat{f}(\hat{x}(k), \hat{w}(k), \tilde{\varsigma}(k))=\Pi_{x}(\tilde{f}(\hat{x}(k), \hat{w}(k), \tilde{\varsigma}(k))),
$$

where $\Pi_{x}: X \rightarrow \hat{X}$ is the map that assigns to any $x \in X$, the representative point $\bar{x} \in \hat{X}$ of the corresponding partition set containing $x$. Abstraction map $\Pi_{x}$ employed in (20) satisfies

$$
\left\|\Pi_{x}(x)-x\right\| \leq \bar{\delta}, \forall x \in X
$$

where $\bar{\delta}$ is the state discretization parameter defined as $\bar{\delta}:=$ $\sup \left\{\left\|x-x^{\prime}\right\|, x, x^{\prime} \in \mathrm{X}_{i}, i=1,2, \ldots, n_{x}\right\}$.

\section{B. Incrementally Stable Nonlinear Stochastic Systems}

In this subsection, we focus on the general setting of discrete-time nonlinear stochastic systems. The FPSF from $\widehat{\Sigma}_{i}$ to $\Sigma_{i}$ is established here under the assumption that the auxiliary system $\Sigma_{\text {auxi }}$ is incrementally ISS as the following definition.

Definition 5.1: A dt-SS $\Sigma_{\text {auxi }}$ is called incrementally input-to-state stable if there exists a function $S_{i}: X_{i} \times$ $X_{i} \rightarrow \mathbb{R}_{\geq 0}$ such that $\forall x_{i}:=x_{i}(k), x_{i}^{\prime}:=x_{i}^{\prime}(k) \in X_{i}$, $\forall w_{i}:=w_{i}(k), w_{i}^{\prime}:=w_{i}^{\prime}(k) \in \tilde{W}_{i}$, the inequalities

$$
\underline{\alpha}_{i}\left(\left\|x_{i}-x_{i}^{\prime}\right\|\right) \leq S_{i}\left(x_{i}, x_{i}^{\prime}\right) \leq \bar{\alpha}_{i}\left(\left\|x_{i}-x_{i}^{\prime}\right\|\right) \text {, }
$$

$$
\begin{aligned}
& \left.\underset{\mathbb{E}}{\operatorname{and}} S_{i}\left(\tilde{f}_{i}\left(x_{i}, w_{i}, \tilde{\varsigma}_{i}\right), \tilde{f}_{i}\left(x_{i}^{\prime}, w_{i}^{\prime}, \tilde{\varsigma}_{i}\right)\right) \mid x_{i}, x_{i}^{\prime}, w_{i}, w_{i}^{\prime}\right]-S_{i}\left(x_{i}, x_{i}^{\prime}\right) \\
& \quad \leq-\bar{\kappa}_{i}\left(S_{i}\left(x_{i}, x_{i}^{\prime}\right)\right)+\bar{\rho}_{\mathrm{int} i}\left(\left\|w_{i}-w_{i}^{\prime}\right\|\right),
\end{aligned}
$$

hold for some $\underline{\alpha}_{i}, \bar{\alpha}_{i}, \bar{\kappa}_{i} \in \mathcal{K}_{\infty}$, and $\bar{\rho}_{\text {int } i} \in \mathcal{K}_{\infty} \cup\{0\}$.

Under Definition 5.1, the next theorem shows a relation between $\Sigma_{i}$ and $\widehat{\Sigma}_{i}$ via establishing an FPSF from $\widehat{\Sigma}_{i}$ to $\Sigma_{i}$.

Theorem 5.2: Let $\Sigma_{\mathrm{aux} i}$ be an incrementally input-to-state stable dt-SS via a function $S_{i}$ as in Definition 5.1, and $\widehat{\Sigma}_{\text {auxi }}$ be its finite MC as constructed in Subsection V-A with state discretization parameter $\bar{\delta}_{i}$. Assume that there exists a function $\gamma_{i} \in \mathcal{K}_{\infty}$ such that

$$
S_{i}\left(x_{i}, x_{i}^{\prime}\right)-S_{i}\left(x_{i}, x_{i}^{\prime \prime}\right) \leq \gamma_{i}\left(\left\|x_{i}^{\prime}-x_{i}^{\prime \prime}\right\|\right), \forall x_{i}, x_{i}^{\prime}, x_{i}^{\prime \prime} \in X_{i} .
$$

Then $S_{i}$ is an FPSF from $\widehat{\Sigma}_{i}$ to $\Sigma_{i}$.

In the following, we impose conditions on the infinite dt-SS $\Sigma_{i}$ in order to find classic pseudo-simulation functions from $\widehat{\Sigma}_{i}$ to $\Sigma_{i}$ for a particular class of nonlinear stochastic systems with an incremental quadratic constraint on the nonlinearity. 


\section{Stochastic Systems with Incremental Quadratic Con- straint on Nonlinearity}

The class of discrete-time nonlinear stochastic systems, considered here, is given by

$$
x_{i}(k+1)=A_{i} x_{i}(k)+E_{i} \varphi_{i}\left(k, F_{i} x_{i}(k)\right)+D_{i} \mathrm{w}_{i}(k)+R_{i} \varsigma_{i}(k),
$$

where the additive noise $\varsigma_{i}(k)$ is a sequence of independent random vectors with multivariate standard normal distributions. Moreover, the time-varying nonlinearity is the one discussed in [25], which satisfies an incremental quadratic inequality: for all $Q_{i} \in \mathcal{Q}_{i}$, where $\mathcal{Q}_{i}$ is the set of symmetric matrices referred to incremental multiplier matrices, the following incremental quadratic constraint holds for all $k \in \mathbb{N}$, and $d_{1}, d_{2} \in \mathbb{R}:$

$$
\left[\begin{array}{c}
d_{2}-d_{1} \\
\varphi_{i}\left(k, d_{2}\right)-\varphi_{i}\left(k, d_{1}\right)
\end{array}\right]^{T} Q_{i}\left[\begin{array}{c}
d_{2}-d_{1} \\
\varphi_{i}\left(k, d_{2}\right)-\varphi_{i}\left(k, d_{1}\right)
\end{array}\right] \geq 0 .
$$

To facilitate subsequent analysis, we write matrix $Q_{i}$ in the following conformal partitioned form

$$
Q_{i}=\left[\begin{array}{ll}
Q_{11 i} & Q_{12 i} \\
Q_{12 i}^{T} & Q_{22 i}
\end{array}\right] .
$$

Remark 5.3: As discussed in [25], the time-varying nonlinearity proposed in (26) is more general that the one presented in [16, Subsection 5.2]. For instance, one can readily recover the slope restriction in [16, Subsection 5.2] for $\varphi_{i}(k, x)=\sin (x), \forall k \in \mathbb{N}$, by considering $Q_{11 i}=$ $1, Q_{12 i}=0, Q_{22 i}=-1$.

We use the tuple

$$
\Sigma_{i}=\left(A_{i}, D_{i}, E_{i}, F_{i}, R_{i}, \varphi_{i}\right),
$$

to refer to the class of nonlinear stochastic systems of the form (25).

Now we provide conditions under which a candidate $S_{i}$ is a classic pseudo-simulation function facilitating the construction of an abstraction $\widehat{\Sigma}_{i}$. To do so, we nominate the following quadratic function

$$
S_{i}\left(x_{i}, \hat{x}_{i}\right)=\left(x_{i}-\hat{x}_{i}\right)^{T} \tilde{M}_{i}\left(x_{i}-\hat{x}_{i}\right),
$$

where $\tilde{M}_{i}$ is a positive-definite matrix of an appropriate dimension. In order to show that $S_{i}$ in (27) is a classic pseudo-simulation function from $\widehat{\Sigma}_{i}$ to $\Sigma_{i}$, we require the following assumption on $\Sigma_{i}$.

Assumption 3: Assume that for some constants $0<\hat{\kappa}_{i}<$ 1 , and $\pi_{i}>0$, there exists the matrix $\tilde{M}_{i}$ of an appropriate dimension such that the following inequality holds:

$$
\begin{aligned}
& {\left[\begin{array}{cc}
\left(1+2 / \pi_{i}\right) A_{i}^{T} \tilde{M}_{i} A_{i} & A_{i}^{T} \tilde{M}_{i} E_{i} \\
E_{i}^{T} \tilde{M}_{i} A_{i} & \left(1+2 / \pi_{i}\right) E_{i}^{T} \tilde{M}_{i} E_{i}
\end{array}\right]} \\
& \preceq\left[\begin{array}{cc}
\hat{\kappa}_{i} \tilde{M}_{i}-F_{i}^{T} Q_{11 i} F_{i} & -F_{i}^{T} Q_{12 i} \\
-Q_{12 i}^{T} F_{i} & -Q_{22 i}
\end{array}\right] .
\end{aligned}
$$

Now, we raise the main result of this subsection.

Theorem 5.4: Assume $\Sigma_{i}=\left(A_{i}, D_{i}, E_{i}, F_{i}, R_{i}, \varphi_{i}\right)$ satisfies Assumption 3. Let $\widehat{\Sigma}_{i}$ be its finite $\mathrm{MC}$ as described in Subsection V-A but for the original system with the state discretization parameter $\bar{\delta}_{i}$. Then function $S_{i}$ defined in (27) is a classic pseudo-simulation function (with $M=1$ ) from $\widehat{\Sigma}_{i}$ to $\Sigma_{i}$.

\section{CAse Study}

In this section, we demonstrate the effectiveness of the proposed results by considering an interconnected system composed of four discrete-time linear stochastic subsystems, i.e. $\Sigma=\mathcal{I}\left(\Sigma_{1}, \Sigma_{2}, \Sigma_{3}, \Sigma_{4}\right)$, such that one of them is not stable. The stochastic subsystems are given by

$$
\Sigma_{i}:\left\{\begin{array}{l}
x_{1}(k+1)=1.001 x_{1}(k)+0.5 \mathrm{w}_{1}(k)+\varsigma_{1}(k), \\
x_{2}(k+1)=-0.95 x_{2}(k)-0.08 \mathrm{w}_{2}(k)+\varsigma_{2}(k), \\
x_{3}(k+1)=-0.94 x_{3}(k)-0.05 \mathrm{w}_{3}(k)+\varsigma_{3}(k), \\
x_{4}(k+1)=0.6 x_{4}(k)+0.9 \mathrm{w}_{4}(k)+\varsigma_{4}(k),
\end{array}\right.
$$

where

$$
\mathbf{w}_{1}=x_{2}+x_{3}, \mathbf{w}_{2}=x_{1}+x_{3}, \mathbf{w}_{3}=x_{2}, \mathbf{w}_{4}=x_{3},
$$

with sets $X_{i}=\left[\begin{array}{ll}0 & 0.1\end{array}\right]$, and $W_{i}=\left[\begin{array}{ll}0 & 0.2\end{array}\right], \forall i \in\{1,2,3,4\}$. As seen, the first subsystem is not stable. Then we proceed with looking at the solution of $\Sigma_{i}$ two steps ahead, i.e., $M=$ 2 ,

$$
\Sigma_{\mathrm{aux} i}:\left\{\begin{array}{l}
x_{1}(k+2)=0.962 x_{1}(k)+\tilde{D}_{1} w_{1}(k)+\tilde{R}_{1} \tilde{\varsigma}_{1}(k), \\
x_{2}(k+2)=0.8665 x_{2}(k)+\tilde{D}_{2} w_{2}(k)+\tilde{R}_{2} \tilde{\varsigma}_{2}(k), \\
x_{3}(k+2)=0.8876 x_{3}(k)+\tilde{D}_{3} w_{3}(k)+\tilde{R}_{3} \tilde{\varsigma}_{3}(k), \\
x_{4}(k+2)=0.36 x_{4}(k)+\tilde{D}_{4} w_{4}(k)+\tilde{R}_{4} \tilde{\varsigma}_{4}(k),
\end{array}\right.
$$

where

$$
\begin{aligned}
& \tilde{D}_{1}=[0.0005 ;-0.0095]^{T}, \tilde{D}_{2}=[-0.0041 ; 0.1112]^{T}, \\
& \tilde{D}_{3}=[0.004 ; 0.0945]^{T}, \tilde{D}_{4}=[-0.045 ;-0.306]^{T}, \\
& w_{1}=\left[x_{2} ; x_{3}\right], w_{2}=\left[x_{1} ; x_{3}\right], w_{3}=\left[x_{1} ; x_{2}\right], w_{4}=\left[x_{2} ; x_{3}\right], \\
& \tilde{\varsigma}_{1}(k)=\left[\varsigma_{3}(k) ; \varsigma_{2}(k) ; \varsigma_{1}(k) ; \varsigma_{1}(k+1)\right], \tilde{\varsigma}_{3}(k)=\left[\varsigma_{2}(k) ; \varsigma_{3}(k) ; \varsigma_{3}(k+1)\right], \\
& \tilde{\varsigma}_{2}(k)=\left[\varsigma_{3}(k) ; \varsigma_{1}(k) ; \varsigma_{2}(k) ; \varsigma_{2}(k+1)\right], \tilde{\varsigma}_{4}(k)=\left[\varsigma_{3}(k) ; \varsigma_{4}(k) ; \varsigma_{4}(k+1)\right] .
\end{aligned}
$$

Moreover, $\tilde{R}_{i}=\left[\tilde{R}_{i 1} ; \tilde{R}_{i 2} ; \tilde{R}_{i 3} ; \tilde{R}_{i 4}\right]^{T} \forall i \in\{1,2\}$, where

$$
\begin{aligned}
& \tilde{R}_{11}=0.5, \tilde{R}_{12}=0.5, \tilde{R}_{13}=1.001, \tilde{R}_{14}=1, \\
& \tilde{R}_{21}=-0.08, \tilde{R}_{22}=-0.08, \tilde{R}_{23}=-0.95, \tilde{R}_{24}=1 .
\end{aligned}
$$

and $\tilde{R}_{i}=\left[\tilde{R}_{i 1} ; \tilde{R}_{i 2} ; \tilde{R}_{i 3}\right]^{T} \forall i \in\{3,4\}$, where

$$
\tilde{R}_{31}=-0.05, \tilde{R}_{32}=-0.94, \tilde{R}_{33}=1, \tilde{R}_{41}=0.9, \tilde{R}_{42}=0.6, \tilde{R}_{43}=1 \text {. }
$$

As seen, $\tilde{A}_{1}$ is now stable. Now we proceed with constructing the finite $\mathrm{MC}$ from the $M$-sampled system as acquired in (30). We fix FPSF as $S_{i}\left(x_{i}, \hat{x}_{i}\right)=\left\|x_{i}-\hat{x}_{i}\right\|$. One can readily verify that $S_{i}$ is an FPSF from $\widehat{\Sigma}_{i}$ to $\Sigma_{i}$ satisfying condition (8) with $\alpha_{i}(s)=s, \forall i \in\{1,2,3,4\} \forall s \in \mathbb{R}_{\geq 0}$, and condition (9) with

$$
\begin{aligned}
& \kappa_{i}(s)=0.99 s, \forall i \in\{1,2,3,4\}, \rho_{\mathrm{int} 1}(s)=0.8838 s, \\
& \rho_{\text {int } 2}(s)=0.9676 s, \rho_{\mathrm{int} 3}(s)=0.9936 s, \rho_{\mathrm{int} 4}(s)=0.9659 s, \\
& \psi_{1}=36.47 \bar{\delta}_{1}, \psi_{2}=56.85 \bar{\delta}_{2}, \psi_{3}=60.61 \bar{\delta}_{3}, \psi_{4}=3.09 \bar{\delta}_{4} .
\end{aligned}
$$

Now we check small-gain condition (17) that is required for the compositionality result. By taking $\sigma_{i}(s)=s \forall i \in$ $\{1,2,3,4\}$, one can readily verify that the small-gain condition (17) and as a result condition (18) are satisfied. Hence, $V(x, \hat{x})=\max _{i}\left\|x_{i}-\hat{x}_{i}\right\|$ is an FSF from $\widehat{\Sigma}$ to $\Sigma$ satisfying conditions (10) and (11) with $\alpha(s)=s, \kappa(s)=0.99 s$, $\forall s \in \mathbb{R}_{>0}$, and $\psi=60.61 \bar{\delta}$.

We take the state and internal input discretization parameters as 0.0001 . Hence, we have $n_{x_{i}}=1000$, and $n_{w_{i}}=2000$. By starting the initial states of the interconnected systems $\Sigma$ and $\widehat{\Sigma}$ from $\mathbb{1}_{4}$, and by employing Lemma 3.4, we guarantee that the distance between states of $\Sigma$ and of $\widehat{\Sigma}$ will not exceed $\varepsilon=1$ at times $k=2 j, j=\{0, \ldots, 15\}$ with probability at least $91 \%$, i.e.

$$
\mathbb{P}\left\{\left\|x_{a}(k)-\hat{x}_{\hat{a}}(k)\right\| \leq 1, \forall k=2 j, j=\{0, \ldots, 15\}\right\} \geq 0.91 .
$$

Now we quantify the probabilistic closeness guarantee for the whole state trajectory as proposed in (14). Assumption 1 is satisfied for the original interconnected system with 


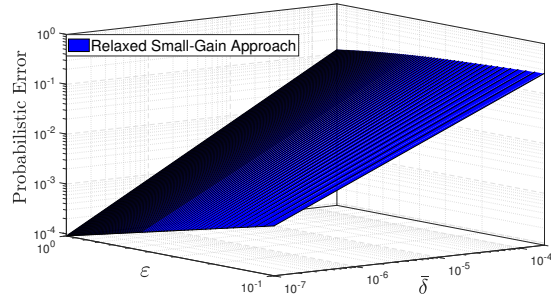

Fig. 1. Error bound proposed in (14) for $M=2$. Plot is in the logarithmic scale for $T_{d}=30$. The probabilistic closeness guarantee is improved by either decreasing $\bar{\delta}$ or increasing $\varepsilon$.

$\mu(s)=1.001 s, \forall s \in \mathbb{R}_{\geq 0}$ and $\theta=\bar{\delta}$. Then by employing Theorem 3.6, we guarantee that the distance between states of $\Sigma$ and of $\widehat{\Sigma}$ will not exceed $\varepsilon=1$ for the whole state trajectory within time horizon $[0,30]$ with probability at least $88 \%$, i.e.

$$
\mathbb{P}\left\{\left\|x_{a}(k)-\hat{x}_{\hat{a}}(k)\right\| \leq 1, \forall k \in[0,30]\right\} \geq 0.88 .
$$

In order to have more practical analysis on the proposed closeness guarantee, we plotted the error bound provided in (14) for $M=2$ in terms of the state discretization parameter $\bar{\delta}$ and confidence bound $\varepsilon$ in Figure 1. As seen, the probabilistic closeness guarantee is improved by either decreasing $\bar{\delta}$ or increasing $\varepsilon$.

Now we provide some discussions on the computation time and memory usage in constructing the finite $\mathrm{MC}$ in both monolithic and compositional manners. To do so, we employ software tool FAUST ${ }^{2}$ on a machine with Windows operating system (Intel i7@3.6GHz CPU and $16 \mathrm{~GB}$ of RAM). The monolithic MC would be a matrix with the dimension of $n_{x_{i}}^{4} \times n_{x_{i}}^{4}$ with $n_{x_{i}}=1000$. By allocating 8 bytes for each entry of the matrix to be stored, one needs a memory of roughly $\frac{8 \times 1000^{4} \times 1000^{4}}{10^{9}}=8 \times 10^{15}$ GB for building the finite $\mathrm{MC}$ in the monolithic manner which is impossible in practice. Now, we proceed with the compositional construction of the finite MC proposed in this work for each subsystem. The constructed MC for each subsystem here is a matrix with the dimension of $\left(n_{x_{i}} \times\right.$ $\left.n_{w_{i}}\right) \times n_{x_{i}}$ (with $n_{x_{i}}=1000, n_{w_{i}}=2000$ ) with a memory usage of roughly $\frac{8 \times 1000 \times 2000 \times 1000}{10^{9}}=16 \mathrm{~GB}$ for each MC and $64 \mathrm{~GB}$ for all $4 \mathrm{MCs}$, and the computation time of 112 seconds for constructing each MC. This implementation clearly shows that the proposed compositional approach in this work significantly mitigates the curse of dimensionality problem in constructing finite MCs monolithically.

\section{DISCUSSION}

In this paper, we provided a compositional framework for the construction of finite Markov chains for networks of not necessarily stable discrete-time stochastic systems. First, we developed a new type of small-gain conditions which are less conservative than the existing ones. We then proposed an approach to construct finite MCs for discrete-time nonlinear stochastic systems satisfying a finite-step version of an incremental stability property. We also constructed finite MCs for a particular class of nonlinear stochastic systems whose 1 -step $\delta$-ISS can be easily verified using the semidefinite programming. Finally, we applied our proposed results to a network of four subsystems where one of them was not stable.

\section{REFERENCES}

[1] A. Abate, M. Prandini, J. Lygeros, and S. Sastry, "Probabilistic reachability and safety for controlled discrete time stochastic hybrid systems," Automatica, vol. 44, no. 11, pp. 2724-2734, 2008.

[2] S. Soudjani and A. Abate, "Adaptive and sequential gridding procedures for the abstraction and verification of stochastic processes," SIAM Journal on Applied Dynamical Systems, vol. 12, no. 2, pp. 921 956, 2013.

[3] S. Soudjani, C. Gevaerts, and A. Abate, "FAUST": Formal abstractions of uncountable-state stochastic processes," in TACAS'15, ser. Lecture Notes in Computer Science, 2015, vol. 9035, pp. 272-286.

[4] M. Zamani and A. Abate, "Approximately bisimilar symbolic models for randomly switched stochastic systems," Systems \& Control Letters, vol. 69 , pp. $38-46,2014$

[5] M. Zamani, A. Abate, and A. Girard, "Symbolic models for stochastic switched systems: A discretization and a discretization-free approach," Automatica, vol. 55, pp. 183-196, 2015.

[6] S. Soudjani, A. Abate, and R. Majumdar, "Dynamic Bayesian networks for formal verification of structured stochastic processes," Acta Informatica, vol. 54, no. 2, pp. 217-242, 2017.

[7] A. Lavaei, S. Soudjani, and M. Zamani, "From dissipativity theory, to compositional construction of finite Markov decision processes," in Proceedings of the 21st ACM International Conference on Hybrid Systems: Computation and Control, 2018, pp. 21-30.

[8] A. Lavaei, S. Soudjani, and M. Zamani, "Compositional (in)finite abstractions for large-scale interconnected stochastic systems," arXiv: 1808.00893, August 2018.

[9] A. Lavaei, S. Soudjani, and M. Zamani, "Approximate probabilistic relations for compositional synthesis of stochastic systems," in Proceedings of the Numerical Software Verification, 2019, pp. 101-109, lecture Notes in Computer Science 11652

[10] A. Lavaei, S. Soudjani, and M. Zamani, "Compositional abstractionbased synthesis of general MDPs via approximate probabilistic relations," arXiv:1906.02930, 2019.

[11] A. Lavaei and M. Zamani, "Compositional construction of finite MDPs for large-scale stochastic switched systems: A dissipativity approach," Proceedings of the 15th IFAC Symposium on Large Scale Complex Systems: Theory and Applications, vol. 52, no. 3, pp. 31-36, 2019.

[12] A. Nejati, S. Soudjani, and M. Zamani, "Abstraction-based synthesis of continuous-time stochastic control systems," in Proceedings of the 18th Eorupan Control Conference, 2019, pp. 3212-3217.

[13] D. Aeyels and J. Peuteman, "A new asymptotic stability criterion for nonlinear time-variant differential equations," IEEE Transactions on automatic control, vol. 43, no. 7, pp. 968-971, 1998.

[14] N. Noroozi, R. Geiselhart, L. Grüne, B. S. Rüffer, and F. R. Wirth, "Nonconservative discrete-time ISS small-gain conditions for closed sets," IEEE Transactions on Automatic Control, vol. 63, no. 5, pp. $1231-1242,2018$

[15] N. Noroozi, A. Swikir, F. R. Wirth, and M. Zamani, "Compositional construction of abstractions via relaxed small-gain conditions part ii: discrete case," in 2018 European Control Conference (ECC), 2018 , pp. $1-4$

[16] A. Lavaei, S. Soudjani, and M. Zamani, "Compositional synthesis of large-scale stochastic systems: A relaxed dissipativity approach," Nonlinear Analysis: Hybrid Systems, provisionaly accepted, arXiv:1902.01223v2, 2019

[17] A. Lavaei, S. Soudjani, and M. Zamani, "Compositional synthesis of not necessarily stabilizable stochastic systems via finite abstractions," in Proceedings of the 18th European Control Conference, 2019, pp. $2802-2807$.

[18] A. Lavaei, S. Soudjani, and M. Zamani, "Compositional synthesis of finite abstractions for continuous-space stochastic control systems: A small-gain approach," Proceedings of the 6th IFAC Conference on Analysis and Design of Hybrid Systems, vol. 51, no. 16, pp. 265-270, 2018

[19] A. Lavaei, S. Soudjani, R. Majumdar, and M. Zamani, "Compositional abstractions of interconnected discrete-time stochastic control systems," in Proceedings of the 56th IEEE Conference on Decision and Control, 2017, pp. 3551-3556.

[20] A. Lavaei, S. Soudjani, and M. Zamani, "Compositional construction of infinite abstractions for networks of stochastic control systems," Automatica, vol. 107, pp. 125-137, 2019.

[21] R. Geiselhart, R. H. Gielen, M. Lazar, and F. R. Wirth, "An alternative converse Lyapunov theorem for discrete-time systems," Systems \& Control Letters, vol. 70, pp. 49-59, 2014.

[22] E. Schechter, Handbook of Analysis and its Foundations. San Diego: Academic Press, 1996.

[23] B. S. Rüffer, "Monotone inequalities, dynamical systems, and paths in the positive orthant of euclidean n-space," Positivity, vol. 14, no. 2 , pp. 257-283, 2010.

[24] M. L. Puterman, Markov decision processes: discrete stochastic dynamic programming. John Wiley \& Sons, 2014.

[25] B. Açıkmeşe and M. Corless, "Observers for systems with nonlinearities satisfying incremental quadratic constraints," Automatica, vol. 47, no. 7, pp. 1339-1348, 2011 